\title{
Triterpenoides de frutos de Melia azedarach: su potencial como agentes de control de insectos
}

\author{
Díaz, M. ${ }^{(1,2,3)}$, Rossini, C. ${ }^{(1)}$ \\ (1) Laboratorio de Ecología Química, Facultad de Química, República Oriental del Uruguay - (2) Instituto de Ciencias \\ Agrarias, Consejo Superior de Investigaciones Científicas, Madrid, España - ${ }^{(3)}$ Instituto de Productos Naturales y Agro- \\ biología de Canarias, IPNA-CSIC, España.
}

Contacto: martinadiaz@fq.edu.uy

Recibido: 25/03/2011 - Aprobado: 9/11/2011

\begin{abstract}
$\underline{\text { Resumen }}$
Melia azedarach L. (Meliaceae) es nativa de Asia pero ha sido naturalizada en muchas partes del planeta. Variados compuestos bioactivos estructuralmente complejos fueron aislados de especímenes asiáticos. Este estudio tuvo como objetivo determinar la actividad anti-insecto de extractos orgánicos de frutos de M. azedarach de especímenes uruguayos y elucidar las estructuras de los compuestos activos.

EI fraccionamiento sistemático de extractos etanólicos llevó a la obtención de una fracción rica en limonoides con actividad contra diferentes insectos modelo. Mostró fuerte deterrencia frente a larvas de Spodoptera littoralis (Lepidoptera: Noctuidae) y adultos de Epilachna paenulata (Coleoptera: Coccinellidae) en bioensayos de opción. Además, inhibió el asentamiento de Myzus persicae y Rhopalosiphum padi (Homoptera: Aphididae). Sin embargo, no mostró toxicidad por contacto contra adultos de Nezara viridula (Hemiptera: Pentatomidae).

Dos compuestos nunca antes reportados en esta especie vegetal y tres previamente reportados pero nunca antes testados contra los insectos nombrados fueron aislados de la fracción.

En síntesis, una fracción rica en triterpenoides del extracto etanólico de frutos de M. azedarach mostró potencial variable contra las diferentes especies de insectos plaga modelo, enfatizando así la importancia de testar los potenciales agentes biocontroladores contra diferentes insectos blanco.

Palabras clave: Limonoides, Paraíso, Epilachna paenulata, Myzus persicae, Rhopalosiphum padi, Spodoptera littoralis.
\end{abstract}

\begin{abstract}
Melia azedarach L. (Meliaceae) is native from Asia but it has been naturalized worldwide. Several structurally complex bioactive compounds have been isolated from Asian specimens. This study aimed to determine the anti-insect activity of organic extracts of M. azedarach fruits from Uruguayan populations, and to structurally elucidate the active compounds.

Systematic fractionation of ethanolic extracts led to a triterpenoid-rich fraction, with strong activity against different insect models. For instance, it was strongly deterrent against larvae of Spodoptera littoralis (Lepidoptera: Noctuidae) and adults of Epilachna paenulata (Coleoptera: Coccinellidae) in choice experiments. Besides, the same fraction showed settling inhibition activity on Myzus persicae and Rhopalosiphum padi (Homoptera: Aphididae). Such fraction showed no contact toxicity against adults of Nezara viridula (Hemiptera: Pentatomidae).

Two compounds never reported for this vegetal species and three previously reported but never evaluated against those insect species, were isolated from that fraction.

On the whole, a triterpenoid fraction from the ethanolic extract of fruits of $M$. azedarach has been evaluated on its anti-insect activity, showing variable potential against different insect pest models, emphasizing the value of testing potential biocontrol products against different insect targets.

Keywords: Limonoids, Paraíso, Epilachna paenulata, Myzus persicae, Rhopalosiphum padi, Spodoptera littoralis.
\end{abstract}

\section{Introducción}

El aumento de la población mundial ha generado la necesidad de incrementar los rendimientos de la producción primaria, lo que a su vez ha ocasionado un aumento del uso de plaguicidas convencionales para controlar las especies plagas. Sin dudas, su uso es efectivo en el corto plazo, pero no es sostenible en el largo plazo, debido al desarrollo de mecanismos de resistencia en dichas especies y a los efectos adversos en el medio ambiente, tanto desde el punto de vista biótico como abiótico. Por estos motivos existe actualmente una tendencia, aun en la producción convencional, a utilizar alternativas a los plaguicidas de síntesis (como parte de lo que se denomina mundialmente Manejo
Integrado de Plagas). Asimismo, se ha intensificado paulatinamente el uso de prácticas alternativas de producción, tales como la llamada "producción orgánica", en la que se tiende a adoptar medidas de control amigables con el medio ambiente, excluyendo el uso de los plaguicidas sintéticos. Se hace por lo tanto necesario contar con opciones, entre las cuales una posibilidad son los productos naturales derivados de plantas ("plaguicidas botánicos").

El árbol de Paraíso, Melia azedarach L. es nativo de Persia, India y China, pero naturalizado en África, Australia y las Américas. El uso de esta especie ornamental con fines medicinales ha sido previamente estudiado (Chinese Pharmacopeia, 2005; Everist, 1974; Hurst, 1942; Watt et al., 1962). Además, esta especie ha mostrado poseer actividad anti-insecto. Han sido descritas propiedades deterrentes de 
oviposición e ingesta, reducción del crecimiento y toxicidad contra diferentes especies de Lepidópteros, Coleópteros (Carpinella et al., 2006) y Dípteros (Coria et al., 2008), no solamente en sus extractos crudos, sino también de algunos constituyentes puros.

Este estudio tuvo como objetivo determinar la actividad antiinsecto de extractos orgánicos de frutos de $M$. azedarach de especímenes uruguayos y elucidar las estructuras de los compuestos activos.

\section{Materiales y Métodos}

\section{Material vegetal}

Los frutos maduros de $M$. azedarach fueron colectados en Montevideo, Uruguay, en julio de 2008. Fueron secados durante 48 $72 \mathrm{~h} \mathrm{a} 40{ }^{\circ} \mathrm{C}$ antes de la molienda y extracción.

\section{Extracción y aislamiento}

Los frutos secos $(716 \mathrm{~g})$ fueron molidos y extraídos con hexano en caliente $(1300 \mathrm{~mL}$, temperatura ambiente, $\mathrm{x} 3)$ antes de la maceración etanólica (1300 mL etanol, temperatura ambiente, $24 \mathrm{~h}$, x 3). El extracto etanólico de frutos (EE frutos) fue concentrado y disuelto en metanol/agua (95:5), para ser particionado con hexano y luego acetato de etilo (AcOEt). El extracto AcOEt $(8,8 \mathrm{~g})$ fue fraccionado por cromatografía flash, eluyendo con AcOEt, de lo que se obtuvieron 8 fracciones. Las fracciones 2 y $3(4,3 \mathrm{~g})$ fueron sometidas a otra cromatografía flash eluída con cloroformo/acetonitrilo desde 6:1 a 6:2 para obtener 14 fracciones (F1 - F14).

De la fracción $4(0.57 \mathrm{~g})$ fueron aislados: $3(1 \mathrm{mg}), 4(<1 \mathrm{mg})$ y 5 $(<1 \mathrm{mg})$ y de las fracciones 5-7 $(1.14 \mathrm{~g})$ fueron aislados: $1(27 \mathrm{mg})$, $2(3.7 \mathrm{mg}), 5(5 \mathrm{mg})$ y otras fracciones que mostraron ser mezclas de aquellos compuestos, así como los mismos en bajo grado de pureza (ej.: mezcla $5+3$ ); se realizaron sobre todos ellos análisis de Resonancia Magnética Nuclear (RMN) mono y bidireccionales y de Espectrometría de Masas de Alta Resolución (EM).

Asimismo, fue obtenido un extracto etanólico de frutos y fraccionado, siguiendo un protocolo muy similar al anteriormente descripto. En esa instancia se obtuvo un extracto rico en limonoides (E lim); los estudios en TLC, HPLC y RMN mostraron una alta concentración de este tipo de compuestos.

\section{Colonias de insectos}

Los adultos de Epilachna paenulata Germar (Coleoptera: Coccinellidae) (especialista en cucurbitáceas) fueron mantenidos sobre plantas de zapallo (Cucurbita pepo L.) bajo condiciones controladas de temperatura $\left(20 \pm 2{ }^{\circ} \mathrm{C}\right)$ y fotoperíodo $(14 \mathrm{~L}: 10 \mathrm{O})$ (Luz:Oscuridad) (Bellomo et al., 2009). En el caso de Nezara viridula (Hemiptera: Pentatomidae), ninfas y adultos fueron mantenidas por separado, alimentados con chauchas y germinadores de lentejas, a $25 \pm 1{ }^{\circ} \mathrm{C},>70 \%$ humedad relativa ambiente y con un fotoperíodo controlado (16L:8O). Ambos insectos fueron criados y utilizados en el Laboratorio de Ecología Química, Uruguay.

Las larvas de Spodoptera littoralis Boisduval (Lepidoptera: Noctuidae) (generalista) fueron mantenidas con dieta artificial (Poitout et al., 1974) a $25 \pm 1{ }^{\circ} \mathrm{C},>70 \%$ humedad relativa ambiente y fotoperíodo controlado (16L:8O). Los adultos de Rhopalosiphum padi L. (Hemiptera: Aphididae) (especialista en gramíneas) y de Myzus persicae Sulzer (Hemiptera: Aphididae) (generalista) fueron mantenidos sobre plantas de Hordeum vulgare L. a $20 \pm 1{ }^{\circ} \mathrm{C} \mathrm{y}$ Capsicum annuum L. a $25 \pm 1{ }^{\circ} \mathrm{C}$, respectivamente. Ambas colonias a $>70 \%$ humedad relativa ambiente y con fotoperíodo controlado (16L:8O). Estos tres insectos fueron mantenidos y utilizados en Instituto de Ciencias Agrarias de Madrid, España.

\section{Bioensayos}

Los bioensayos con insectos masticadores (E. paenulata y $S$. littoralis) se realizaron en placas de Petri $(9 \mathrm{~cm} \times 1 \mathrm{~cm})$ cubiertas con agar (2\%). A los insectos se les ofreció, luego de hambreados por $3 \mathrm{~h}$ y en forma individual, cuatro discos de hojas $(1 \mathrm{~cm} 2)$ de su planta hospedera (C. pepo y $C$. annuum para $E$. paenulata y $S$. littoralis, respectivamente). Dos de los discos (T) se trataron con 100 $\mu \mathrm{g}$ del extracto o $50 \mu \mathrm{g}$ de los productos puros $(10 \mu \mathrm{L}$ de una solución metanólica al $10 \%$ o $5 \%$ ), y los otros dos (C) con $10 \mu \mathrm{L}$ de metanol. Se realizaron 10-15 réplicas para adultos de E. paenulata $(5$ a 10 días de edad) y 6 réplicas para larvas L4 de $S$. littoralis (cada réplica consistió en una placa con un insecto y los cuatro discos de hojas). Se registraron visualmente -en intervalos de 1/16- el área consumida de discos en cada placa y luego fue calculado un Índice de Preferencia usando la fórmula: $\mathrm{IP}=(\mathrm{C}-\mathrm{T}) /(\mathrm{C}+\mathrm{T})$, donde $\mathrm{C}$ y $\mathrm{T}$ son las cantidades consumidas de control y tratado (Bellomo et al., 2009) tras $3 \mathrm{~h}$ de ensayo o cuando había sido consumido el $75 \%$ de uno de los casos.

Los bioensayos con áfidos (M. persicae y $R$. padi, 20 réplicas de 10 áfidos cada una por especie de insecto) fueron realizados en cajas $(3 \times 3 \times 1.5 \mathrm{~cm})$ cubiertas con agar $(2 \%)$ que contenían dos trozos de hojas $(1 \mathrm{~cm} 2)$ de las plantas hospederas $(C$. annuum y $H$. vulgare para $M$. persicae y $R$. padi, respectivamente) sobre una de las cuales se había aplicado previo al ensayo el extracto (T, $100 \mu \mathrm{g} / \mathrm{cm} 2$ ), y sobre la otra la misma cantidad de disolvente (metanol, $10 \mu \mathrm{L}, \mathrm{C}$ ). En cada caja se colocaron 10 áfidos, y se registró el porcentaje de áfidos asentados en la superficie de cada hoja luego de $24 \mathrm{~h}$, de acuerdo a lo reportado en trabajos anteriores (Castillo et al., 2009; Guitiérrez et al., 1997). "Asentados" fueron posados quietos sobre las hojas. Dado el tamaño tan pequeño de estos insectos no se puede constatar visualmente si se están alimentando o no, pero se sabe que si se posan es porque han pasado por las primeras etapas de selección de la comida, y que una vez posados (cuando la detección periférica ha funcionado como elemento decisorio positivo) el siguiente paso corresponde al comienzo de los comportamientos estereotipados que llevan a la ingesta. Un Índice de Preferencia se calculó como IP $=(\% \mathrm{C}-\% \mathrm{~T}) /(\% \mathrm{C}+\% \mathrm{~T})$, donde $\% \mathrm{~T}$ y \% C son los porcentajes de áfidos asentados en las superficies $\mathrm{T}$ y $\mathrm{C}$.

El bioensayo de toxicidad contra chinches fue realizado en viales cilíndricos de vidrio cuyas paredes fueron empapadas con las soluciones etanólicas a testar $(\mathrm{N}=5$ para cada caso), siguiendo las directivas de "The Insecticide Resistance Action Committee" (IRAC, 2006). Luego de evaporado el disolvente se introdujo un adulto en cada vial y se registró la sobrevivencia de los mismos a las 24 y 48 h. Se utilizó viales empapados en etanol como control negativo y viales empapados $\left(6,3 \times 10^{-3} \mathrm{mg} / \mathrm{cm}^{2}\right)$ en solución de Engeo $247 \mathrm{C}$ (Thiametoxam $14 \mathrm{~g}$ - Lambdacihalotrina 14:11 \% p/v) como control positivo. Fueron testadas varias concentraciones de EE frutos y $\mathrm{E}$ lim; $25 \mathrm{mg} / \mathrm{cm}^{2}$ y $0,6 \mathrm{mg} / \mathrm{cm}^{2}$ fueron las mayores cantidades testadas en uno y otro caso.

\section{Análisis estadísticos}

Los datos obtenidos de los bioensayos fueron analizados por Test de Rangos de Wilcoxon (nivel de significancia: $p<0,05$ ). En el caso de los ensayos con áfidos, las réplicas donde menos de 5 áfidos se asentaban no fueron consideradas.

\section{Resultados y Discusión}

En la Figura 1 se muestran las estructuras elucidadas de los compuestos aislados ( 1 a 5 ) del extracto etanólico de frutos de $M$. azedarach. 


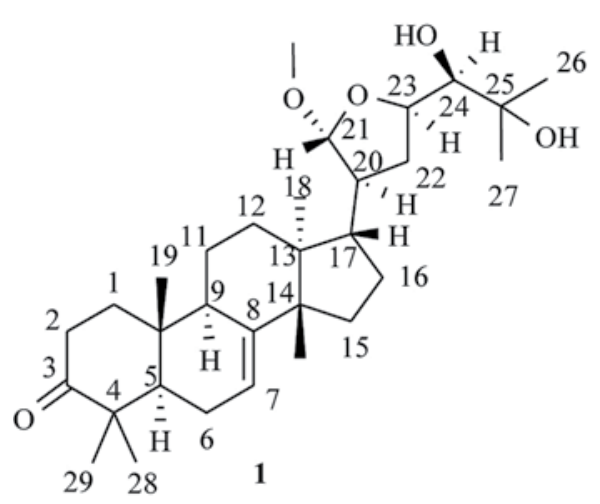

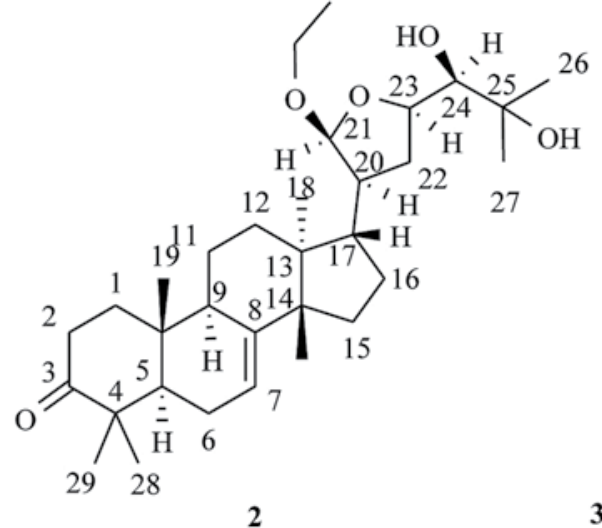

2

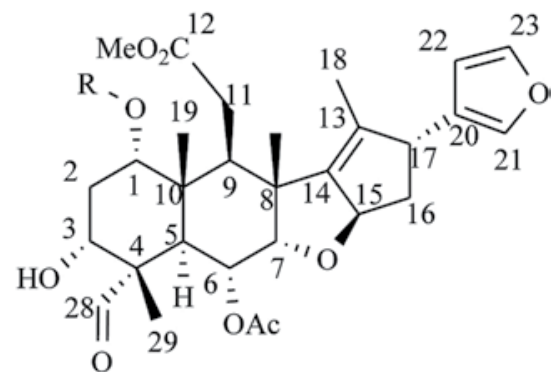

$3 \mathrm{R}=$ cinnamoilo; $4 \mathrm{R}=$ benzoilo; $5 \mathrm{R}=$ tigloilo

Figura 1. Compuestos aislados del árbol del Paraíso: 1:21 $\alpha$-metilmelianodiol; 2: 21ß-etilmelianodiol; 3: 1-O-Detigloyl-1-O-cinnamoylohchinolal; 4: 1-O-Detigloyl-1-O-benzoylohchinolal; 5: Ohchinolal.

En la Tabla 1 se muestran los resultados de actividad del extracto etanólico bruto, el extracto rico en limonoides y los compuestos puros testados.

\begin{tabular}{|c|c|c|c|c|}
\hline \multicolumn{5}{|c|}{ Especies de insectos } \\
\hline \multicolumn{5}{|c|}{ Índice de preferencia $(\mathrm{IP} \pm \mathrm{ES})$} \\
\hline & M. persicae & R.padi & S. littoralis & E. paenulata \\
\hline EE frutos & $0,3 \pm 0,1^{*}$ & $0,1 \pm 0,1$ & $0,8 \pm 0,1^{*}$ & $0,3 \pm 0,3$ \\
\hline$E \lim$ & $0,5 \pm 0,1^{*}$ & $0,19 \pm 0,06 *$ & $1,00 \pm 0,00^{*}$ & $0,98 \pm 0,02 *$ \\
\hline 1 & $-0,2 \pm 0,1$ & $0,1 \pm 0,1$ & $0,3 \pm 0,2$ & $0,1 \pm 0,4$ \\
\hline 3 & $0,2 \pm 0,1$ & $-0,06 \pm 0,09$ & $0,3 \pm 0,2$ & $0,9 \pm 0,1^{*}$ \\
\hline 5 & $-0,2 \pm 0,2$ & $0,1 \pm 0,1$ & $0,93 \pm 0,07^{*}$ & $0,7 \pm 0,2 *$ \\
\hline $5+3$ & $-0,1 \pm 0,1$ & $0,1 \pm 0,1$ & $0,92 \pm 0,04^{*}$ & $1,0 \pm 0,0 *$ \\
\hline
\end{tabular}

Tabla 1. Actividad del extracto bruto, E lim y compuestos aislados contra los insectos testados.

Los estudios de RMN y EM revelaron que 1 correspondía a 21 $\alpha$-metilmelianodiol, el análogo metilado de melianodiol, compuesto completamente elucidado estructuralmente por Puripattanavon et al. (2000), cuando lo aisló de la meliácea Aglaia andamanica. 1 fue aislado, previo a este trabajo, de Cedrela silensis (Meliaceae) (Mitsui et al., 2007), así como de Poncirus trifoliata (Rutaceae) (Xu, 2008), pero nunca de nuestro árbol del Paraíso. Asimismo, 2 resultó ser un compuesto con un esqueleto principal idéntico a 1 pero que presenta un sustituyente etoxilo en lugar de metoxilo en la posición 21 , orientado éste en posición $\beta$ en lugar de $\alpha: 21 \beta$-etilmelianodiol. 2 tampoco había sido antes reportado para esta especie vegetal. El análisis de los espectros de RMN y MS reveló que los compuestos 3-5 resultaron ser: 1-O-Detigloyl-1-O-cinnamoylohchinolal, 1-O-Detigloyl-1-O-benzoylohchinolal y ohchinolal, los cuales habían sido aislados de este árbol anteriormente (Zhou et al., 2004; Fukuyama et al., 1983).

El extracto bruto de frutos de Paraíso mostró deterrencia frente a ambos insectos generalistas, pero fue inactivo frente a los especialistas (Tabla 1). Dadas las diferencias en hábitos alimenticios de insectos especialistas y generalistas, con frecuencia se espera que los generalistas muestren mayor tolerancia a metabolitos secundarios de plantas que los especialistas. En el caso de este extracto, al ser testado contra los cuatro insectos modelo, se observó lo contrario. Una explicación probable para este hecho es que los insectos especialistas a lo largo de la evolución no han desarrollado receptores que les indiquen el estímulo negativo que significan estos metabolitos secundarios para los generalistas. El extracto de limonoides mostró mayor actividad que el crudo frente a los generalistas y además fue activo contra los especialistas, revelando deterrencia absoluta contra E. paenulata. Los limonoides son compuestos que se encuentran presentes en varias especies de Meliaceas y han demostrado poseer actividad anti insecto (Murray, 2001); es esperable, entonces, que un extracto rico en este tipo de productos naturales sea más activo que el extracto bruto. Ni el EE frutos ni E lim mostraron toxicidad contra los adultos de $N$. viridula (resultados no mostrados).

Los compuestos 1,3 y 5 resultaron ser inocuos frente a los áfidos cuando se testaron individualmente, pero algunos de ellos retuvieron la actividad contra los insectos masticadores. Este fue el caso de 3 y 5; 3 fue activo solamente frente al especialista, aunque mostrando actividad al ser testado como mezcla 3/5 (1:1). Esto y la fuerte actividad de E lim expresan que puede existir cierta sinergia entre estos compuestos frente a la acción contra los insectos testados. Asimismo, la inocuidad frente a los áfidos puede deberse a que el estímulo negativo que necesitan este tipo de insectos para rechazar el alimento se encuentre en algún otro paso del complejo proceso de selección del hospedero que involucra tanto estímulos químicos como físicos (Powel et al., 2006), que no están siendo investigados en los bioensayos realizados en este estudio.

\section{Conclusiones}

En síntesis, 1, 2, 3, 4 y 5 fueron aislados del extracto de frutos de Paraísos locales ( 1 y 2 aislados por primera vez de esta especie vegetal), presentando diferentes patrones de actividad contra insectos modelo que, en su mayoría, habían mostrado sensibilidad a un extracto rico en todos estos compuestos juntos. Estos compuestos no habían sido testados nunca antes contra los insectos utilizados en este trabajo. Asimismo, el extracto bruto de frutos de este árbol presentó actividad frente a dos de los insectos testados.

\section{Reconocimientos}

Agradecemos a Eduardo Alonso Paz, Gustavo Seoane, Roberto Zoppolo, Facundo Ibáñez, Azucena González Coloma, Ricardo Guillermo Álvarez y Carmen Elisa Díaz Hernández por su asesoría técnica en diversas materias; a PEDECIBA-Química y los convenios LATU-Facultad de Química y CSIC, España-UdelaR, por diversos financiamientos para llevar adelante estos estudios. 


\section{Referencias}

- BELLOMO, A.; CAMARANO, S.; ROSSINI, C.; GONZALEZ, D. Enantiospecific synthesis and insect feeding activity of sulfur-containing cyclitols. En: Carbohydrate Research. 2009, 344(1):44-51.

- CARPINELLA, M.; DEFAGO, M.; VALLADARES, G.; PALACIOS, S. Role of Melia azedarach L (Meliaceae) for the control of insects and acari: present status and future prospects. En: CARPINELLA, M. (ed). Naturally occuring bioactive compounds. London: Elsevier, 2006.

- CASTILLO, L.; GONZALEZ-COLOMA, A.; GONZALEZ, A.; DIAZ, M.; SANTOS, E.; ALONSO-PAZ, E.; BASSAGODA M. J.; ROSSINI, C. Screening of Uruguayan plants for deterrent activity against insects. En: Industrial Crops and Products. 2009, 29:235-240.

- CHINESE PHARMACOPEIA COMMITTEE. Chinese Pharmacopeia. Beijing: Chemical Industry Press, 2005. Vol. 3, p. 248.

- CORIA, C.; ALMIRON, W.; VALLADARES, G.; CARPINELLA C.; LUDUENA, F.; DEFAGO, M.; PALACIOS, S. Larvicide and oviposition deterrent effects of fruit and leaf extracts from Melia azedarach L. on Aedes aegypti (L.) (Diptera : Culicidae). En: Bioresource Technology. 2008, 99(8):3066-3070.

- EVERIST, S. L. Poisonous Plants of Australia. Sydney: Angus and Robertson, 1974. p. 368

- FUKUYAMA,Y.; MIURA, I.; OCHI, M. Bitter limonoids from the fruit of Melia azedarch L. var. japonica Makino. En: Bulletin of the Chemical Society of Japan. 1983, 56:1139-1142.

- GUTIÉRREZ, C.; FERRERES, A.; REINA, M.; CABRERA, R.; GONZÁLEZ-COLOMA, A. Behavioral and sub-lethal effects of structurally related lower terpenes on Myzus persicae. En: Journal of Chemical Ecology. 1997, 23:1641-1650.

- HURST, E., Poisonous plants of New South Wales. Sydney: [s.n.], 1942. p 214.

- IRAC. Insecticide Resistance Action Committee. [En línea]. [s.1.]: [s.n.], 2006. [Consulta: mayo de 2011]. Disponible en: http://www.irac-online. org/

- MITSUI, K.; SAITO, H.; YAMAMURA, R.; FUKAYA, H.; HITOTSUYANAGI,Y.; TAKEYA, K. Apotirucallane and tirucallane triterpenoids from Cedrela sinensis. En: Chemical \& Pharmaceutical Bulletin. 2007, 55:1442-1447.

- MURRAY, B. I.; Biopesticides based on phytochemicals. En: Koul, O.; Dhaliwal, G. S. Phytochemical Biopesticides. [s.1.]: [s.n.], 2001. p 1.

- POITOUT, S.; BUES, R. Rearing larvae of twentyeight species of Noctuidae and two species of Arctiidae (Lepidoptera) on a simple artificial diet breeding peculiarities according to the different species. En: Annales de Zoologie Ecologie Animale. 1974, 6(3):431-441.

- POWELL, G.; TOSH, C.; HARDIE, J. Host plant selection by aphids: behavioural, evolutionary and applied perspectives. En: Annu. Rev. Entomol. 2006, 51:309-330.

- WATT, J. M.; BREYER-BRANDWICK, M. G. The Medicinal and Poisonous Plants of Southern and Eastern Africa. 2nd ed. London: [s.n.], 1962.

- XU, G.; KIM, J.; KIM, S.Y.; RYU, J.; KIM, Y.; JUNG, S. Terpenoids and coumarins isolated from the fruits of Poncirus trifoliata. En: Chemical \& Pharmaceutical Bulletin. 2008, 56:839-842.

- ZHOU, H.L.; HAMAZAKI, A.; FONTANA, J.D.; TAKAHASHI, H.; ESUMI, T.; WANDSCHEER, C.B. New ring C-seco limonoids from Brazilian Melia azedarach and their cytotoxic activity. En: Journal of Natural Products. 2004, 67:1544-1547. 